\title{
The NQ01 Pro187Ser polymorphism and breast cancer susceptibility: evidence from an updated meta-analysis
}

Qiliu Peng ${ }^{1}$, Yu Lu ${ }^{1}$ Xianjun Lao ${ }^{1}$, Zhiping Chen ${ }^{2}$, Ruolin $\mathrm{Li}^{3}$, Jingzhe Sui ${ }^{1}$ Xue Qin ${ }^{1 *}$ and Shan $\mathrm{Li}^{1}$

\begin{abstract}
Background: $\mathrm{NAD}(\mathrm{P}) \mathrm{H}$ : quinone oxidoreductase 1 (NQO1) plays a central role in catalyzing the two-electron reduction of quinoid compounds into hydroquinones. The NQO1 Pro187Ser polymorphism was found to correlate with a lower enzymatic activity, which may result in increased incidence of carcinomas including breast cancer. Previous studies investigating the association between NQO1 Pro187Ser polymorphism and breast cancer risk showed inconsistent results. We performed a meta-analysis to summarize the possible association.
\end{abstract}

Methods: All studies published from January 1966 to February 2014 on the association between NQO1 Pro1875er polymorphism and breast cancer risk were identified by searching electronic databases PubMed, EMBASE, Cochrane library, and Chinese Biomedical Literature database (CBM). The association between NQO1 Pro187Ser polymorphism and breast cancer risk was assessed by odds ratios (ORs) together with their 95\% confidence intervals (Cls).

Results: Ten studies with 2,773 cases and 4,076 controls were finally included in the meta-analysis. We did not observe a significant association between NQO1 Pro1875er polymorphism and breast cancer risk when all studies were pooled into the meta-analysis. In subgroup analysis by ethnicity, significant increased breast cancer risk was found in Caucasians (Ser/Pro vs. Pro/Pro: $\mathrm{OR}=1.145,95 \% \mathrm{Cl}=1.008-1.301, \mathrm{P}=0.038$; Ser/Ser + Ser/Pro vs. Pro/Pro: $\mathrm{OR}=1.177,95 \% \mathrm{Cl}=1.041-1.331, \mathrm{P}=0.009)$. When stratified by source of control, significant increased breast cancer risk was found in population-based studies (Ser/Pro vs. Pro/Pro: $\mathrm{OR}=1.180,95 \% \mathrm{Cl}=1.035-1.344, \mathrm{P}=0.013$; Ser/Ser + Ser/Pro vs. Pro/Pro: $\mathrm{OR}=1.191,95 \% \mathrm{Cl}=1.050-1.350, \mathrm{P}=0.007)$. However, in subgroup analyses according to menopausal status, quality score, and HWE in controls, no any significant association was detected.

Conclusions: Our meta-analysis provides the evidence that the NQO1 Pro187Ser polymorphism contributed to the breast cancer susceptibility among Caucasians. Further large and well-designed studies are needed to confirm this association.

Virtual slides: The virtual slide(s) for this article can be found here: http://www.diagnosticpathology.diagnomx.eu/ vs/1248639991252504

Keywords: NQO1, Polymorphism, Breast cancer, Meta-analysis

\footnotetext{
*Correspondence: qinxue919@126.com

'Department of Clinical Laboratory, First Affiliated Hospital of Guangxi

Medical University, Nanning, Guangxi 530021, China

Full list of author information is available at the end of the article
} 


\section{Background}

Breast cancer is the most common cancer and the second most common cause of cancer-related death in women. In 2008 there were 182,460 women diagnosed with breast cancer, and 40,480 women died of this disease [1]. In several developing countries, such as China, breast cancer has surpassed cervical cancer and become the leading cause of cancer death among females [2]. Though the exact mechanism of breast carcinogenesis is still unclear, it has been well accepted that oxidative stress resulting from excess reactive oxygen species and deficiency in antioxidant capabilities play important roles in breast cancer etiology [3,4].

$\mathrm{NAD}(\mathrm{P}) \mathrm{H}$ :quinone oxidoreductase 1 (NQO1), also known as diphtheria toxin diaphorase (DT-diaphorase), is a cytosolic flavoenzyme which is present in human epithelial and endothelial tissues. NQO1 is considered as an anticancer enzyme because it protect cells from oxidative damage by preventing quinones from entering the oneelectron reduction which is catalyzed by cytochrom b5 reductase or $\mathrm{P} 450$ reductase to generate semiquinone free radicals and reactive oxygen species [5]. On the contrary, with its unique property of transferring two electrons at a time by using either NADH or NADPH as reducing cofactor, NQO1 catalyze quinones and quinine-imines into hydroquinones, which are thought less toxic and easier to excrete when conjugated [6,7]. The NQO1 gene, mapped to chromosome 16q22.1, is $17.2 \mathrm{~kb}$ in length and contains 6 exons and 5 introns [8]. There were at least 270 SNPs in the NQO1 gene according to the dbSNP database (http:// www.ncbi.nlm.nih.gov/SNP), including the most commonly occurring C-to- $\mathrm{T}$ transition at nucleotide position 609 in exon 6 (rs1800566, 609C $>\mathrm{T})$, which results in a proline-to-serine amino-acid substitution at codon 187 (Pro187Ser) in the protein. It was reported that the variant T allele was associated with reduced NQO1 enzymatic activity in both human cell lines and primary human tissues $[9,10]$. Furthermore, there is a clear allele dosage effect of the NQO1 609 T genotypes on NQO1 enzymatic activity, with the variant homozygotes (TT) having the lowest, the heterozygotes (CT) having the intermediate, and the wildtype homozygotes $(\mathrm{CC})$ having the highest NQO1 enzyme activity [11-13]. Given that the previous studies have consistently shown that the variant $\mathrm{T}$ allele resulted in reduced enzymatic activity, it was biologically reasonable to hypothesize a potential relationship between the NQO1 Pro187Ser polymorphism and cancer susceptibility.

In the past two decades, a number of molecular epidemiological studies have evaluated the association between the NQO1 Pro187Ser polymorphism and breast cancer risk, but the results remain inconsistent. Several studies have previously suggested that the NQO1 Pro187Ser polymorphism was associated with an increased risk of breast cancer $[14,15]$. However, other studies have failed to confirm such an association $[3,16]$. In addition, a meta-analysis by Yuan et al. [17] found that the NQO1 Pro187Ser polymorphism may contribute to breast cancer development in Caucasians. However, evidence was limited because only 6 studies were available at that time. In addition, only ethnicity was considered in the subgroup analysis and the source of heterogeneity was not explored in this study. As some new studies emerging $[16,18,19]$, to provide the most comprehensive assessment of the associations between the NQO1 Pro187Ser polymorphism and breast cancer risk, we performed an updated meta-analysis of all available studies with extensive exploration of the source of heterogeneity and subgroup analyses.

\section{Methods}

\section{Search strategy}

We conducted a comprehensive literature search in PubMed, EMBASE, Cochrane library, and Chinese Biomedical Literature (CBM) databases form January 1966 to February 2014 using the following search strategy: ("breast cancer") and ("NAD(P)H:quinone oxidoreductase 1", or "NQO1"). There was no restriction on sample size, population, language, or type of report. All eligible studies were retrieved and their references were checked for other relevant studies. The literature retrieval was performed in duplication by two independent reviewers (Qiliu Peng and $\mathrm{Yu} \mathrm{Lu}$ ). When multiple publications reported on the same or overlapping data, we chose the most recent or largest population. When a study reported the results on different subpopulations, we treated it as separate studies in the meta-analysis.

\section{Inclusion and exclusion criteria}

Studies included in the meta-analysis were required to meet the following criteria: (1) case-control or cohort studies which evaluated the association between NQO1 Pro187Ser polymorphism and breast cancer risk; (2) used an unrelated case-control design; (3) had an odds ratio (OR) with 95\% confidence interval (CI) or other available data for estimating OR (95\% CI); and (4) the control population did not contain malignant tumor patients. Studies were excluded if one of the following existed: (1) no control population; (2) duplicate of previous publication; and (3) insufficient information for data extraction.

\section{Data extraction}

Two authors (Qiliu Peng and Xue Qin) independently reviewed and extracted data from all eligible studies. Data extracted from eligible studies included the first author, year of publication, ethnicity, country of origin, genotyping method, source of control, matching criteria, breast cancer ascertainment, total numbers of cases and controls 
and genotype frequencies of cases and controls. Ethnic backgrounds were categorized as Caucasian, Asian, and Arab. When a study did not state the ethnic descendent or if it was impossible to separate participants according to such phenotype, the group reported was termed as "mixed ethnicity". To ensure the accuracy of the information extracted, the two authors checked the data extraction results and reached consensus on all of the items. If different results generated, they would check the data again and have a discussion to come to an agreement. If these two authors could not reach a consensus, another author (Shan $\mathrm{Li}$ ) was consulted to resolve the dispute and a final decision was made by the majority of the votes. Menopausal status was divided into premenopausal and postmenopausal and was additionally recorded for stratified analysis.

\section{Quality score evaluation}

The quality of eligible studies was evaluated independently by two authors (Qiliu Peng and $\mathrm{Yu} \mathrm{Lu}$ ) according to a set of predefined criteria (Table 1) based on the scale of Thakkinstian et al. [20]. The revised criteria cover the source of controls, representativeness of cases, ascertainment of breast cancer, total sample size, quality control of genotyping methods, and Hardy-Weinberg equilibrium (HWE) in the control population. Discrepancies were resolved by consensus. Scores ranged from 0 (lowest) to 10 (highest). Articles with scores equal to or higher than 7 were considered "high-quality" studies, whereas those with scores less than 7 were considered "low-quality" studies.

\section{Statistical analysis}

The strength of the association between NQO1 Pro187Ser polymorphism and breast cancer risk was assessed by odds ratios (ORs) with 95\% confidence intervals (CIs). The significance of the pooled OR was determined by a $\mathrm{Z}$ test and the $\mathrm{p}$ value less than 0.05 was considered significant. The association of NQO1 Pro187Ser polymorphism with breast cancer risk was assessed using codominant model (Ser/Ser vs. Pro/Pro and Ser/Pro vs. Pro/ Pro), recessive model (Ser/Ser vs. Ser/Pro + Pro/Pro), and dominant model (Ser/Ser + Ser/Pro vs. Pro/Pro).

The $\chi^{2}$ based $Q$ test was used to assess the heterogeneity among studies [21,22]. If the result of the $Q$ test was $P_{Q}<0.1$, indicating the presence of heterogeneity, a random-effects model (the DerSimonian and Laird method) was used to estimate the summary ORs [21]; otherwise, when the result of the $Q$ test was $P_{Q} \geq 0.1$, indicating the absence of heterogeneity, the fixed-effects model (the Mantel-Haenszel method) was used [22]. To explore the sources of heterogeneity among studies, we performed logistic metaregression and subgroup analyses. The following study characteristics were included as covariates in
Table 1 Scale for quality assessment

\begin{tabular}{lc}
\hline Criteria & Score \\
\hline Representativeness of cases & 2 \\
Selected from cancer registry or multiple cancer center sites & 1 \\
Selected from oncology department or cancer institute & 0 \\
Selected without clearly defined sampling frame or with & \\
extensive inclusion/exclusion criteria & \\
Source of controls & 2 \\
Population or community based & 1.5 \\
Both population-based and hospital-based/healthy volunteers/ & \\
blood donors & 1 \\
Hospital-based controls without breast cancer & 0.5 \\
Cancer-free controls without total description & 0 \\
Not described & \\
Ascertainment of breast cancer & 2 \\
Histological or pathological confirmation & 1 \\
Diagnosis of breast cancer by patient medical record & 0.5 \\
Not described & 0 \\
Sample size & \\
>1000 & \\
200-1000 & \\
<200 & \\
Quality control of genotyping methods & \\
Clearly described a different genotyping assay to confirm & 1 \\
Not described & \\
Hardy-Weinberg equilibrium & \\
Hardy-Weinberg equilibrium in controls & 1 \\
\hline
\end{tabular}

the metaregression analysis: genotyping methods (PCRRFLP vs. not PCR-RFLP), ethnicity (Caucasians vs. not Caucasians), source of controls (Hospital-based vs. Population-based), quality scores (High-quality vs. Low-quality), HWE status (Yes vs. No), and breast cancer ascertainment (pathologically or histologically confirmed vs. other diagnosis criteria). Subgroup analyses were conducted by ethnicity, menopausal status, quality score, source of control, and HWE in controls. Galbraith plots analysis was performed for further exploration of the heterogeneity.

Sensitivity analysis was performed by sequential removal of individual studies. Publication bias was evaluated using a funnel plot and Egger's regression asymmetry test. The distribution of the genotypes in the control population was tested for HWE using a goodness-of-fit $\chi^{2}$ test. All analyses were performed using Stata software, version 12.0 (Stata Corp., College Station, TX). 


\section{Result}

\section{Study characteristics}

Base on our search criterion, 112 individual records were found, but only 11 full-text publications were preliminarily identified for further detailed evaluation. According to the exclusion criteria, 2 publications were excluded including 1 provide insufficient information for data extraction [23], and 1 was a meta-analysis [17]. Manual search of references cited in the published studies did not reveal any additional articles. As a result, a total of 9 relevant studies met the inclusion criteria for the meta-analysis [3,14-16,18,19,24-26]. Among them, one of the eligible studies contained data on two ethnic groups [15], and we treated it independently. Therefore, a total of 10 separate comparisons including 2,773 breast cancer cases and 4,076 controls were finally included in our meta-analysis. The main characteristics of the studies are presented in Table 2. Of all the eligible studies, 6 were conducted in Caucasian populations, 3 were in Asians, and 1 was in Arabs. Six studies were population-based and 4 were hospital-based studies. All studies used validated methods including PCR-RFLP, PCR-SSCP, PCR-CTPP, TaqMan assay, and 5' exonuclease assay to genotype the NQO1 Pro187Ser polymorphism. The breast cancer cases were histologically or pathologically confirmed in 7 of the eligible studies. The genotype distributions of the controls in 2 studies were not consistent with HWE [16,25].

\section{Meta-analysis}

The results of meta-analysis of the association between NQO1 Pro187Ser polymorphism and breast cancer risk were shown in Table 3. We did not observe a significant association between the NQO1 Pro187Ser polymorphism and breast cancer risk when all studies were pooled into the meta-analysis (Ser/Ser vs. Pro/Pro: OR $=1.251,95 \% \mathrm{CI}$ 0.843-1.856, $P=0.266$; Ser/Pro vs. Pro/Pro: $\mathrm{OR}=1.015$, 95\% CI 0.860-1.198, $P=0.860 ;$ Ser/Ser + Ser/Pro vs. Pro/ Pro: $\mathrm{OR}=1.058,95 \%$ CI $0.899-1.245, P=0.498$; Ser $/$ Ser vs. Ser/Pro + Pro/Pro: OR =1.317, 95\% CI 0.919-1.887, $P=0.133)$. In subgroup analysis by ethnicity, statistical significant increased breast cancer risk was found in Caucasians (Ser/Pro vs. Pro/Pro: $\mathrm{OR}=1.145$, 95\% CI = 1.008-1.301, $\mathrm{P}=0.038 ;$ Ser/Ser + Ser/Pro vs. Pro/Pro: $\mathrm{OR}=1.177,95 \% \mathrm{CI}=1.041-1.331, \mathrm{P}=0.009$; Figure 1 ), but not in Asians and Arabs. In stratified analysis by source of control, significant increased breast cancer risk was also found in population-based studies (Ser/Pro vs. Pro/Pro: $\mathrm{OR}=1.180,95 \% \mathrm{CI}=1.035-1.344, \mathrm{P}=0.013$; Ser/ Ser + Ser/Pro vs. Pro/Pro: $\mathrm{OR}=1.191,95 \%$ CI $=1.050-$ $1.350, \mathrm{P}=0.007$; Figure 2), but not in hospital-based studies. However, when stratified by menopausal status, quality score, and HWE in controls, statistical significant association was not detected in all subgroups.

\section{Test of heterogeneity}

Statistical significant heterogeneity among studies was observed when all studies were pooled into the metaanalysis (Ser/Ser vs. Pro/Pro: $P_{Q}=0.004 ;$ Ser/Pro vs. Pro/ Pro: $P_{Q}=0.033$; Ser $/$ Ser + Ser $/$ Pro vs. Pro/Pro: $P_{Q}=0.023$; Ser/Ser vs. Ser/Pro + Pro/Pro: $P_{Q}=0.007$; Table 3$)$. To explore the sources of heterogeneity, we performed metaregression and subgroup analyses. Metaregression analysis of data showed that the genotyping methods, source of controls, ethnicity, quality scores, HWE status, and breast cancer ascertainment were not effect modifiers of heterogeneity. Subgroup analyses stratified by ethnicity,

Table 2 Characteristics of studies included in this meta-analysis

\begin{tabular}{|c|c|c|c|c|c|c|c|c|c|}
\hline $\begin{array}{l}\text { First author } \\
\text { (Year) }\end{array}$ & Country & Ethnicity & $\begin{array}{l}\text { Genotyping } \\
\text { methods }\end{array}$ & $\begin{array}{l}\text { Matching } \\
\text { criteria }\end{array}$ & $\begin{array}{l}\text { Sample size } \\
\text { (case/control) }\end{array}$ & $\begin{array}{l}\text { Source of } \\
\text { control }\end{array}$ & $\begin{array}{c}\mathrm{BC} \\
\text { ascertainment }\end{array}$ & $\begin{array}{l}\text { Quality } \\
\text { scores }\end{array}$ & $\begin{array}{c}\text { HWE } \\
(P \text { value })\end{array}$ \\
\hline Yao 2013 & China & Asian & TaqMan Assay & Age & $162 / 190$ & $\mathrm{HB}$ & PC & 7 & 0.540 \\
\hline Menzel Tyrol 2004 & Austria & Caucasian & $\begin{array}{c}\text { 5' exonuclease } \\
\text { assay }\end{array}$ & Region & $218 / 424$ & PB & $\mathrm{HC}$ & 7.5 & 0.175 \\
\hline $\begin{array}{c}\text { Menzel Prague } \\
2004\end{array}$ & Czech & Caucasian & $\begin{array}{c}\text { 5' exonuclease } \\
\text { assay }\end{array}$ & Age, region & $190 / 231$ & PB & $\mathrm{HC}$ & 7 & 0.650 \\
\hline $\begin{array}{l}\text { Siegelmann-Danieli } \\
2002\end{array}$ & America & Caucasian & PCR-SSCP & Age, region & $346 / 235$ & PB & $\mathrm{HC}$ & 6 & 0.869 \\
\hline Singh 2010 & India & Asian & PCR-RFLP & $\begin{array}{l}\text { Age, smoking, } \\
\text { region }\end{array}$ & $200 / 200$ & $\mathrm{HB}$ & Other & 5.5 & 0.000 \\
\hline Hamajima 2002 & Japan & Asian & PCR-CTPP & NA & $237 / 640$ & $\mathrm{HB}$ & $\mathrm{HC}$ & 6 & 0.046 \\
\hline Sarmanova 2004 & Czech & Caucasian & PCR-RFLP & Age, ethnicity & $238 / 310$ & $\mathrm{HB}$ & $\mathrm{HC}$ & 6 & 0.576 \\
\hline Aston 2005 & America & Caucasian & PCR-RFLP & Age, region & $564 / 1212$ & PB & Other & 8.5 & 0.549 \\
\hline Hong 2007 & America & Caucasian & TaqMan Assay & Age, ethnicity & $496 / 495$ & PB & Other & 7 & 0.531 \\
\hline Lajin 2013 & Syria & Arab & ARMS-PCR & Ethnicity & $122 / 139$ & PB & $\mathrm{HC}$ & 7.5 & 0.253 \\
\hline
\end{tabular}

BC, Breast cancer; HC, Histologically confirmed; PC, Pathologically confirmed; NA, Not available; PB, Population-based; HB, Hospital-based; HWE, Hardy-Weinberg equilibrium in control population; PCR-RFLP, Polymerase chain reaction-restriction fragment length polymorphism; PCR-SSCP, Polymerase chain reaction-single strand conformation polymorphism; PCR-CTPP, polymerase chain reaction with confronting two-pair primers; ARMS-PCR, amplification refractory mutation system-PCR. 
Table 3 Meta-analysis of NQO1 Pro187Ser polymorphism and breast cancer risk

\begin{tabular}{|c|c|c|c|c|c|c|c|c|c|}
\hline \multirow[t]{2}{*}{ Analysis } & \multirow[t]{2}{*}{$\begin{array}{l}\text { No. of } \\
\text { studies }\end{array}$} & \multicolumn{2}{|c|}{ Ser/Ser vs. Pro/Pro (Homozygote) } & \multicolumn{2}{|c|}{ Ser/Pro vs. Pro/Pro (Heterozygote) } & \multicolumn{2}{|c|}{$\begin{array}{l}\text { Ser/Ser + Ser/Pro vs. Pro/Pro } \\
\text { (Dominant model) }\end{array}$} & \multicolumn{2}{|c|}{ Ser/Ser vs. Ser/Pro + Pro/Pro (Recessive model } \\
\hline & & OR $(95 \% \mathrm{Cl})$ & $P / P_{Q}$ & OR $(95 \% \mathrm{Cl})$ & $P / P_{Q}$ & OR $(95 \% \mathrm{Cl})$ & $P / P_{Q}$ & OR $(95 \% \mathrm{Cl})$ & $P / P_{Q}$ \\
\hline Overall & 10 & $1.251(0.843-1.856)$ & $0.266 / 0.004$ & $1.015(0.860-1.198)$ & $0.860 / 0.033$ & $1.058(0.899-1.245)$ & $0.498 / 0.023$ & $1.317(0.919-1.887)$ & $0.133 / 0.007$ \\
\hline \multicolumn{10}{|l|}{ Ethnicity } \\
\hline Caucasian & 6 & $1.725(0.884-3.368)$ & $0.110 / 0.003$ & $1.145(1.008-1.301)$ & $0.038 / 0.505$ & $1.177(1.041-1.331)$ & $0.009 / 0.358$ & $1.654(0.847-3.232)$ & $0.141 / 0.003$ \\
\hline Asian & 3 & $0.785(0.571-1.080)$ & $0.137 / 0.676$ & $0.926(0.771-1.124)$ & $0.309 / 0.225$ & $0.941(0.790-1.129)$ & $0.310 / 0.536$ & $1.008(0.765-1.329)$ & $0.955 / 0.195$ \\
\hline Arab & 1 & $1.362(0.525-3.533)$ & $0.526 /-$ & $1.168(0.687-1.986)$ & $0.565 /-$ & $1.202(0.731-1.976)$ & $0.469 /-$ & $1.290(0.506-3.286)$ & $0.594 /-$ \\
\hline \multicolumn{10}{|l|}{ Menopausal status } \\
\hline Premenopausal & 2 & $1.124(0.681-1.853)$ & $0.648 / 0.299$ & $1.110(0.900-1.368)$ & $0.330 / 0.431$ & $1.104(0.902-1.352)$ & $0.337 / 0.604$ & $1.160(0.719-1.871)$ & $0.544 / 0.147$ \\
\hline Postmenopausal & 2 & $0.637(0.356-1.140)$ & $0.129 / 0.833$ & $0.950(0.737-1.225)$ & $0.694 / 0.187$ & $0.918(0.718-1.174)$ & $0.495 / 0.113$ & $0.762(0.445-1.305)$ & $0.322 / 0.482$ \\
\hline \multicolumn{10}{|l|}{ Quality score } \\
\hline$\geq 7$ & 6 & $1.287(0.746-2.221)$ & $0.364 / 0.112$ & $1.062(0.864-1.307)$ & $0.567 / 0.376$ & $1.107(0.897-1.365)$ & $0.343 / 0.147$ & $1.315(0.817-2.117)$ & $0.260 / 0.231$ \\
\hline$<7$ & 4 & $1.251(0.627-2.496)$ & $0.524 / 0.021$ & $0.942(0.698-1.271)$ & $0.694 / 0.070$ & $0.983(0.738-1.309)$ & $0.906 / 0.071$ & $1.368(0.701-2.669)$ & $0.358 / 0.015$ \\
\hline \multicolumn{10}{|l|}{ Source of control } \\
\hline $\mathrm{HB}$ & 4 & $1.099(0.604-2.001)$ & $0.757 / 0.017$ & $0.966(0.823-1.140)$ & $0.311 / 0.301$ & $0.912(0.744-1.125)$ & $0.179 / 0.252$ & $1.344(0.784-2.304)$ & $0.283 / 0.015$ \\
\hline PB & 6 & $1.419(0.802-2.511)$ & $0.230 / 0.124$ & $1.180(1.035-1.344)$ & $0.013 / 0.782$ & $1.191(1.050-1.350)$ & $0.007 / 0.385$ & $1.335(0.767-2.323)$ & $0.308 / 0.230$ \\
\hline \multicolumn{10}{|l|}{ HWE in controls } \\
\hline Yes & 8 & $1.450(0.878-2.395)$ & $0.147 / 0.004$ & $1.080(0.906-1.287)$ & $0.389 / 0.069$ & $1.136(0.964-1.340)$ & $0.128 / 0.079$ & $1.463(0.931-2.299)$ & $0.099 / 0.011$ \\
\hline No & 2 & $0.791(0.539-1.162)$ & $0.232 / 0.378$ & $0.800(0.609-1.050)$ & $0.108 / 0.389$ & $0.788(0.609-1.021)$ & $0.091 / 0.607$ & $0.926(0.656-1.308)$ & $0.663 / 0.105$ \\
\hline
\end{tabular}

$P_{Q} P$ values of Q-test for heterogeneity test. OR, odds ratio; $\mathrm{Cl}$, confidence intervals; $\mathrm{HB}$, Hospital-based studies; $\mathrm{PB}$, Population-based studies; $\mathrm{HWE}$, Hardy-Weinberg equilibrium. 


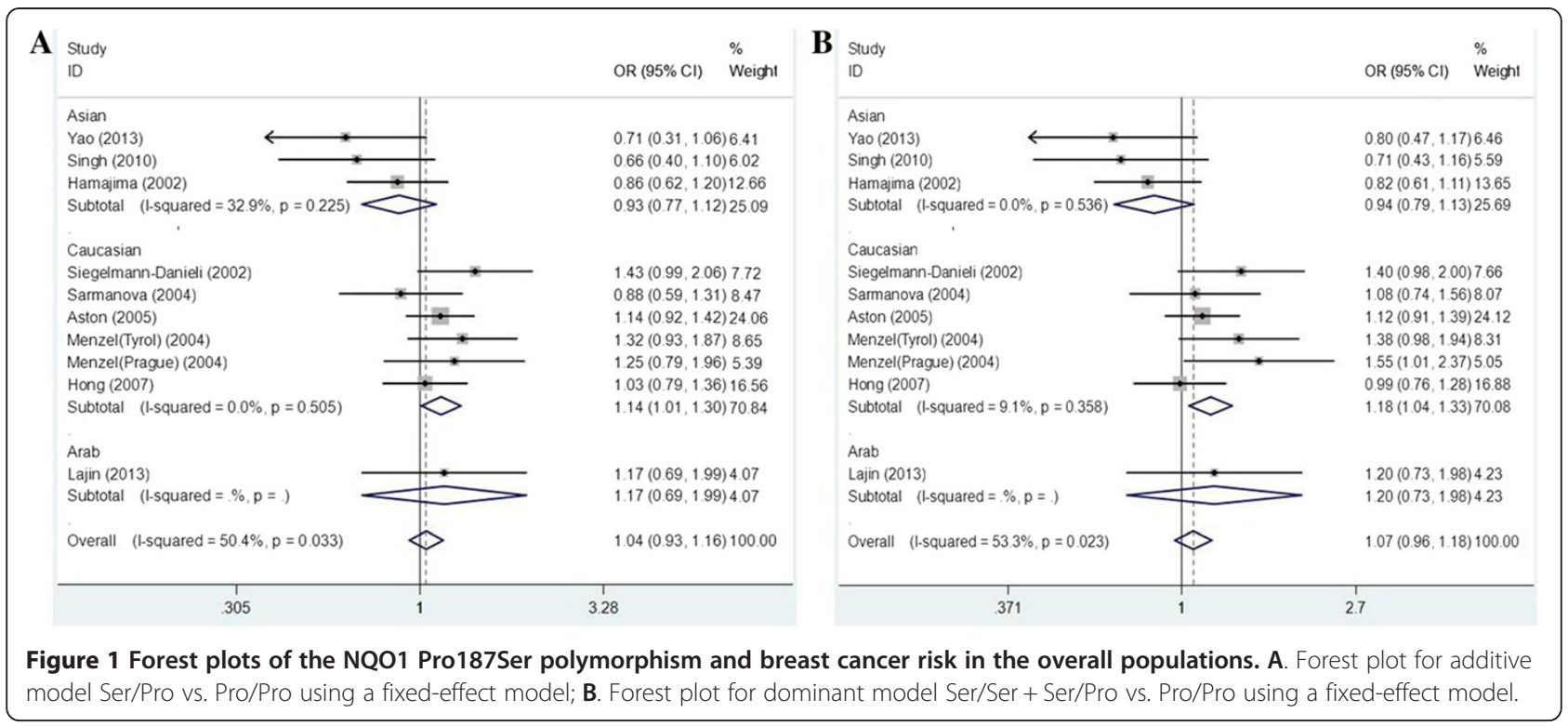

menopausal status, quality score, source of control, and HWE status showed that heterogeneity still existed among Caucasians, low quality studies, population-based studies, and studies consistent with HWE (Table 2). To further explore the source of heterogeneity, we performed Galbraith plots analysis to identify the outliers which might contribute to the heterogeneity. Our results showed that the study Menzel et al. [15] was the outlier in the overall populations. All $P_{Q}$ values were greater than 0.10 after excluding the study Menzel et al. [15] in the overall populations, Caucasians, population-based studies, and studies consistent with HWE. However, the significance of the summary ORs for NQO1 Pro187Ser polymorphism in the overall population and subgroup analyses were not influenced by omitting this study [15].

\section{Sensitivity analysis}

Sensitivity analysis was performed to assess the influence of each individual study on the pooled ORs by sequential removal of individual studies. The results suggested that no individual study significantly affected the pooled ORs. In addition, sensitivity analysis was further performed by omitting the studies by Singh et al. [16] and Hamajima et al. [25] in which the control populations were not in accordance with HWE. The significance of all ORs was not altered after excluding these two studies (data not shown), indicating that our results were robust and reliable.

\section{Publication bias}

Begg's funnel plot and Egger's test were performed to assess the publication bias of literatures in all comparison
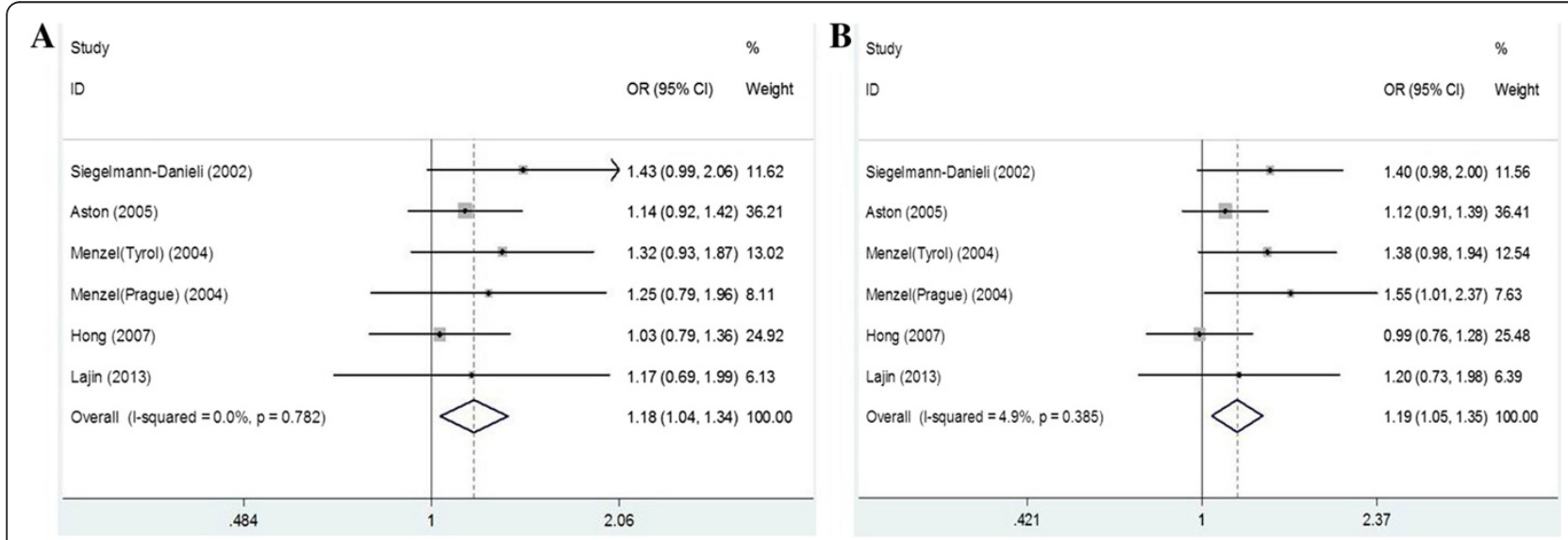

Figure 2 Forest plots of the NQO1 Pro187Ser polymorphism and breast cancer risk in population-based studies. A. Forest plot for additive model Ser/Pro vs. Pro/Pro using a fixed-effect model; B. Forest plot for dominant model Ser/Ser + Ser/Pro vs. Pro/Pro using a fixed-effect model. 
models. The shape of the funnel plot did not reveal any evidence of obvious asymmetry. Then, the Egger's test was used to provide statistical evidence of funnel plot symmetry. The results still did not suggest any evidence of publication bias $(P=0.114$ for $\mathrm{Ser} / \mathrm{Ser}$ vs. Pro/Pro; $P=0.277$ for Ser/Pro vs. Pro/Pro; $P=0.704$ for Ser $/ \mathrm{Ser}+$ Ser/Pro vs. Pro/Pro, Figure 3; $P=0.226$ for Ser/Ser vs. Ser/Pro + Pro/Pro).

\section{Discussion}

Previous studies investigating the associations between NQO1 Pro187Ser polymorphism and breast cancer presented inconsistent results, and most of those studies involved no more than a few hundred breast cancer cases, which is too few to assess any genetic effects reliably. Meta-analysis has been recognized as an important tool to more precisely define the effect of selected genetic polymorphisms on the risk for disease and to identify potential important sources of between-study heterogeneity [27-29]. Hence, we performed this meta-analysis including all available studies to provide the most comprehensive assessment of the associations between the NQO1 Pro187Ser polymorphism and breast cancer risk. Our results showed that the NQO1 is a candidate gene for breast cancer susceptibility. The NQO1 Pro187Ser polymorphism was associated with an increased breast cancer risk among Caucasians (Ser/Pro vs. Pro/Pro: $\mathrm{OR}=1.145,95 \% \mathrm{CI}=$ 1.008-1.301, $\mathrm{P}=0.038 ;$ Ser $/ \mathrm{Ser}+\mathrm{Ser} / \mathrm{Pro}$ vs. Pro/Pro: $\mathrm{OR}=1.177,95 \% \mathrm{CI}=1.041-1.331, \mathrm{P}=0.009)$. Our result is consistent with the previous meta-analysis performed by Yuan et al. [17].

Given the biochemical properties of NQO1 in protecting cells from oxidative damage and tumor development, this result may be biologically plausible. NQO1 is a key enzyme which catalyzes the two-electron reduction of quinoid compounds into hydroquinones, which reduces and detoxifies quinines and thus protects cells against redox cycling and oxidative stress [30,31]. Previous study suggested that some variants, especially those in the promoter regions of genes, may affect either the expression or activity levels of enzymes and therefore may be mechanistically associated with cancer risk [32]. The NQO1 Pro187Ser polymorphism is a C-to-T transition in the NQO1 gene which leads to a proline to serine amino acid substitution at codon 187 in the protein [33,34]. The NQO1 Pro187Ser polymorphism has been found correlated with decreased enzymatic activity of NQO1 and may affect host's susceptibility to cancer by changing the enzymatic activity of NQO1 [33,34]. More importantly, the NQO1 Pro187Ser polymorphism has been shown associated with increased risk for many different types of cancers, including colorectal cancer [35], lung cancer [36], esophageal cancer [37], and hepatocellular carcinoma [38]. Our results add new evidence that the NQO1 Pro187Ser polymorphism contributes to cancer susceptibility.

In subgroup analysis stratified by ethnicity, the NQO1 Pro187Ser polymorphism presented a risk factor for breast cancer in Caucasian populations, but not in Asian and Arab subjects. The inconsistent data among the different ethnicities may indicate different effects of the NQO1 Pro187Ser polymorphism on breast risk in different ethnic genetic and environmental backgrounds. Studies reported that NQO1 enzyme not only detoxify carcinogenic compounds [6] but also bioactivate several kinds of procarcinogen [39]; thus, decreased activity of NQO1 enzyme may have dual effect on carcinogenesis.

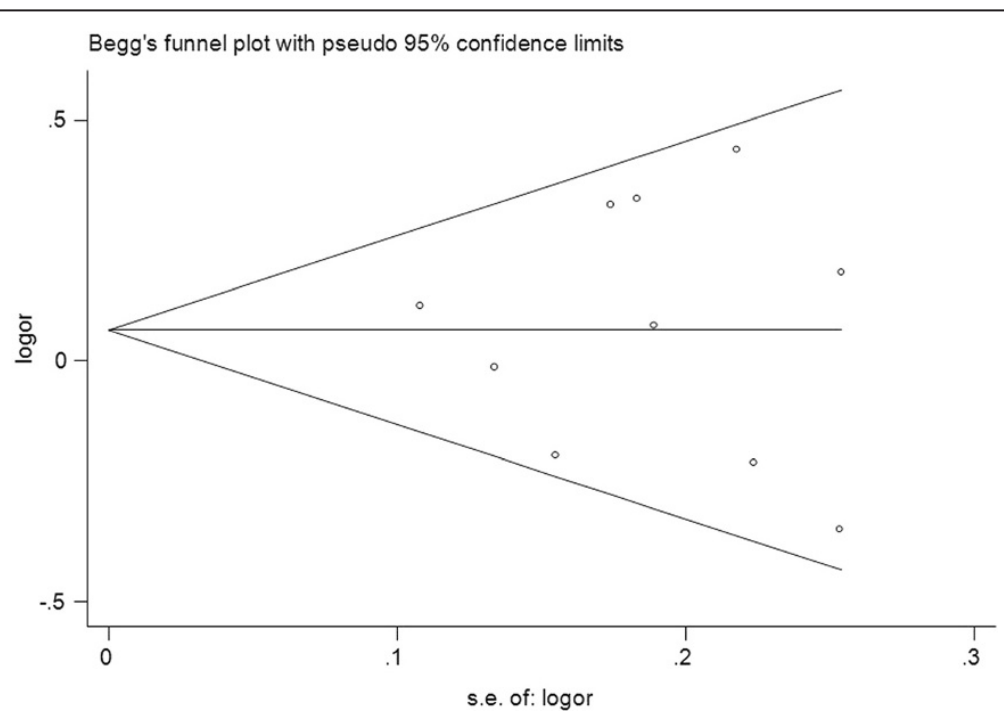

Figure 3 Funnel plots for publication bias of NQO1 Pro187Ser polymorphism and breast cancer risk in the overall populations (dominant model Ser/Ser + Ser/Pro vs. Pro/Pro: $P=0.704)$. 
There may be some alternative ways in the Asian and Arab populations to detoxify carcinogenic compounds which can effectively compensate for the loss of NQO1 enzyme activity. Nevertheless, owing to the limited number of relevant studies among Asian and Arab populations included in the meta-analysis, the observed negative association between NQO1 Pro187Ser polymorphism and breast cancer risk in Asians and Arabs is likely to be caused by chance because study with small sample sizes may have insufficient statistical power to detect a slight effect or may have generated a fluctuated risk estimate. Currently there are only 3 studies in Asian population and 1 in Arab population for NQO1 Pro187Ser polymorphism and breast cancer risk. Therefore, the negative results of the Asain and Arab populations should be interpreted with caution.

In subgroup analysis according to the source of control, statistical significant increased breast cancer risk was found in population-based studies but not in hospitalbased studies. The reason may be that the hospital-based studies have inherent selection biases due to the fact that such controls may not be representative of the study population or the general population, particularly when the genotypes under investigation were associated with the disease-related conditions that hospital-based controls may have. Thus, the use of proper and representative population-based control participants is of great importance in reducing biases in such genotype association studies.

Heterogeneity is a potential problem when interpreting the results of a meta-analysis, and finding the sources of heterogeneity is one of the most important goals of metaanalysis [40,41]. In the present study, statistical significant between-study heterogeneity among studies was observed when all studies were pooled into the meta-analysis (Ser/Ser vs. Pro/Pro: $P_{Q}=0.004 ;$ Ser/Pro vs. Pro/Pro: $P_{Q}=0.033 ;$ Ser/Ser + Ser/Pro vs. Pro/Pro: $P_{Q}=0.023$; Ser/ Ser vs. Ser/Pro + Pro/Pro: $P_{Q}=0.007$; Table 3). To explore the sources of heterogeneity, we performed metaregression and subgroup analyses. Metaregression analysis of data showed that the genotyping methods, ethnicity, source of controls, quality scores, HWE status, and breast cancer ascertainment were not effect modifiers of heterogeneity. Subgroup analyses stratified by ethnicity, menopausal status, quality score, source of control, and HWE status showed that heterogeneity still existed among Caucasians, low quality studies, population-based studies, and studies consistent with HWE (all $P_{Q}$ values $<0.10$ ). Subsequently, we performed Galbraith plots analysis to further explore the source of heterogeneity. Galbraith plots analysis showed that the study Menzel et al. [15] was the outlier in all genetic models in the overall populations. When excluding the study Menzel et al. [15], the heterogeneity decreased obviously and all $P_{Q}$ values were greater than 0.10 in all genetic comparison models in overall populations, Caucasians, population-based studies, and studies consistent with HWE. However, the summary ORs in the overall populations, Caucasians, population-based studies, and studies consistent with HWE were not material changed by omitting this study, indicating that our results were robust and reliable. The results indicated that the study Menzel et al. [15] was the major source of the heterogeneity in the meta-analysis.

However, there are still some limitations in this metaanalysis. First, in subgroup analysis by ethnicity, the included studies regarded only Caucasians, Asians, and Arabs for NQO1 Pro187Ser polymorphism. Data concerning other ethnicities such as Africans were not found. Thus, additional studies are warranted to evaluate the effect of this functional polymorphism on breast cancer risk in different ethnicities, especially in Africans. Second, our results were based on unadjusted estimates. We did not perform analysis adjusted for other covariates such as age, obesity, drinking and smoking status, use of contraceptives, environment factors, and so on, because of the unavailable original data of the eligible studies. Third, subgroup analyses were based on studies with relevant information available. Owing to the lack of detailed information in most studies, the subgroup analysis for menopausal status consisted of only two studies for each subgroup, which might not be sufficient to reach a reliable conclusion.

\section{Conclusions}

This meta-analysis provided a more precise estimation based on larger sample size compared with the individual studies and previous meta-analysis. Our study suggested that NQO1 Pro187Ser polymorphism might contribute to breast cancer risk, especially in Caucasian populations. However, it is necessary to conduct large sample studies using standardized unbiased genotyping methods, homogeneous breast cancer patients, and well-matched controls to further validate the results of our meta-analysis. Moreover, gene-gene and gene-environment interactions should also be considered in the analysis. Such studies taking these factors into account may eventually lead to a better, more comprehensive understanding of the association between NQO1 Pro187Ser polymorphism and breast cancer risk.

\section{Abbreviations \\ HWE: Hardy-Weinberg equilibrium; NQO1: NAD(P)H: quinone oxidoreductase 1; SNP: Single nucleotide polymorphism; OR: Odds ratio; Cl: Confidence interval.}

\section{Competing interest}

The authors declare that they have no competing interests.

\section{Authors' contributions}

QP performed the literature search, data extraction, statistical analysis and drafted the manuscript. $Y L, X L, Z P, R L$, JS, and $X Q$ participated in data 
extraction. SL supervised the literature search, data extraction, statistical analysis and drafted the manuscript. All authors read and approved the final manuscript.

\section{Funding}

The work described in this paper was supported by the National Natural Science Fundation (No. 81260302), Guangxi education and innovation projects for graduate student in 2014 (No. YCBZ2014031).

\section{Author details}

1Department of Clinical Laboratory, First Affiliated Hospital of Guangxi Medical University, Nanning, Guangxi 530021, China. ${ }^{2}$ Department of Occupational Health and Environmental Health, School of Public Health, Guangxi Medical University, Nanning, Guangxi, China. ${ }^{3}$ Department of Medicine Research, First Affiliated Hospital of Guangxi Medical University, Nanning, Guangxi, China.

Received: 27 March 2014 Accepted: 18 May 2014

Published: 29 May 2014

\section{References}

1. Ferlay J, Shin HR, Bray F, Forman D, Mathers C, Parkin DM: Estimates of worldwide burden of cancer in 2008: GLOBOCAN 2008. Int J Cancer 2010, 127:2893-2917.

2. Jemal A, Bray F, Center MM, Ferlay J, Ward E, Forman D: Global cancer statistics. CA Cancer J Clin 2011, 61:69-90.

3. Hong CC, Ambrosone CB, Ahn J, Choi JY, McCullough ML, Stevens VL, Rodriguez C, Thun MJ, Calle EE: Genetic variability in iron-related oxidative stress pathways (Nrf2, NQ01, NOS3, and HO-1), iron intake, and risk of postmenopausal breast cancer. Cancer Epidemiol Biomarkers Prev 2007, 16:1784-1794.

4. Ambrosone CB: Oxidants and antioxidants in breast cancer. Antioxid Redox Signal 2000, 2:903-917.

5. Schlager JJ, Powis G: Cytosolic NAD(P)H: (quinone-acceptor)oxidoreductase in human normal and tumor tissue: effects of cigarette smoking and alcohol. Int J Cancer 1990, 45:403-409.

6. Siegel D, Gustafson DL, Dehn DL, Han JY, Boonchoong P, Berliner L, Ross D: NAD(P)H:quinone oxidoreductase 1: role as a superoxide scavenger. Mol Pharmacol 2004, 65:1238-1247.

7. Winski SL, Koutalos Y, Bentley DL, Ross D: Subcellular localization of NAD (P)H:quinone oxidoreductase 1 in human cancer cells. Cancer Res 2002, 62:1420-1424.

8. Rosvold EA, McGlynn KA, Lustbader ED, Buetow KH: Identification of an $\mathrm{NAD}(\mathrm{P}) \mathrm{H}$ :quinone oxidoreductase polymorphism and its association with lung cancer and smoking. Pharmacogenetics 1995, 5:199-206.

9. Traver RD, Siegel D, Beall HD, Phillips RM, Gibson NW, Franklin WA, Ross D: Characterization of a polymorphism in $\mathrm{NAD}(\mathrm{P}) \mathrm{H}$ : quinone oxidoreductase (DT-diaphorase). Br J Cancer 1997, 75:69-75.

10. Siegel D, McGuinness SM, Winski SL, Ross D: Genotype-phenotype relationships in studies of a polymorphism in $\mathrm{NAD}(\mathrm{P}) \mathrm{H}$ :quinone oxidoreductase 1. Pharmacogenetics 1999, 9:113-121.

11. Moran JL, Siegel D, Ross D: A potential mechanism underlying the increased susceptibility of individuals with a polymorphism in $\mathrm{NAD}(\mathrm{P}) \mathrm{H}$ : quinone oxidoreductase 1 (NQO1) to benzene toxicity. Proc Natl Acad Sci US A 1999, 96:8150-8155.

12. Ross D, Siegel D: NAD(P)H:quinone oxidoreductase 1 (NQO1, DT-diaphorase), functions and pharmacogenetics. Methods Enzymo/ 2004, 382:115-144.

13. Kuehl BL, Paterson JW, Peacock JW, Paterson MC, Rauth AM: Presence of a heterozygous substitution and its relationship to DT-diaphorase activity. Br J Cancer 1995, 72:555-561.

14. Sarmanova J, Susova S, Gut I, Mrhalova M, Kodet R, Adamek J, Roth Z, Soucek P: Breast cancer: role of polymorphisms in biotransformation enzymes. Eur J Hum Genet 2004, 12:848-854

15. Menzel HJ, Sarmanova J, Soucek P, Berberich R, Grunewald K, Haun M, Kraft HG: Association of NQO1 polymorphism with spontaneous breast cancer in two independent populations. Br J Cancer 2004, 90:1989-1994.

16. Singh V, Upadhyay G, Rastogi N, Singh K, Singh MP: Polymorphism of xenobiotic-metabolizing genes and breast cancer susceptibility in North Indian women. Genet Test Mol Biomarkers 2011, 15:343-349.

17. Yuan W, Xu L, Chen W, Wang L, Fu Z, Pang D, Li D: Evidence on the association between NQ01 Pro187Ser polymorphism and breast cancer risk in the current studies: a meta-analysis. Breast Cancer Res Treat 2011, 125:467-472.

18. Yao S, Xu H, Chen Y, Shen X, Ju X, Wu W, Huang H, Dai L: Relationship between genetic polymorphisms of quinone oxidoreductase 1 and susceptibility and chemosensitivity of breast cancer. Chin J Exp Surg 2013, 125:684-687.

19. Lajin B, Alhaj Sakur A, Alachkar A: Association between polymorphisms in apoptotic genes and susceptibility for developing breast cancer in Syrian women. Breast Cancer Res Treat 2013, 138:611-619.

20. Thakkinstian A, McEvoy M, Minelli C, Gibson P, Hancox B, Duffy D, Thompson J, Hall I, Kaufman J, Leung TF, Helms PJ, Hakonarson H, Halpi E, Navon R, Attia J: Systematic review and meta-analysis of the association between \{beta\}2-adrenoceptor polymorphisms and asthma: a HuGE review. Am J Epidemiol 2005, 162:201-211.

21. DerSimonian R, Laird N: Meta-analysis in clinical trials. Control Clin Trials 1986, 7:177-188

22. Mantel N, Haenszel W: Statistical aspects of the analysis of data from retrospective studies of disease. J Natl Cancer Inst 1959, 22:719-748.

23. Fowke JH, Shu XO, Dai Q, Jin F, Cai Q, Gao YT, Zheng W: Oral contraceptive use and breast cancer risk: modification by $\mathrm{NAD}(\mathrm{P}) \mathrm{H}$ : quinone oxoreductase (NQO1) genetic polymorphisms. Cancer Epidemiol Biomarkers Prev 2004, 13:1308-1315.

24. Siegelmann-Danieli N, Buetow $\mathrm{KH}$ : Significance of genetic variation at the glutathione S-transferase $\mathrm{M} 1$ and $\mathrm{NAD}(\mathrm{P}) \mathrm{H}$ :quinone oxidoreductase 1 detoxification genes in breast cancer development. Oncology 2002, 62:39-45.

25. Hamajima N, Matsuo K, Iwata H, Shinoda M, Yamamura Y, Kato T, Hatooka S, Mitsudomi T, Suyama M, Kagami $Y$, Ogura M, Ando M, Sugimura $Y$, Tajima K: NAD(P)H: quinone oxidoreductase 1 (NQ01) C609T polymorphism and the risk of eight cancers for Japanese. Int J Clin Oncol 2002, 7:103-108.

26. Aston CE, Ralph DA, Lalo DP, Manjeshwar S, Gramling BA, DeFreese DC, West AD, Branam DE, Thompson LF, Craft MA, Mitchell DS, Shimasaki CD, Mulvihill $J$, Jupe ER: Oligogenic combinations associated with breast cancer risk in women under 53 years of age. Hum Genet 2005, 116:208-221.

27. Ma L, Zhao J, Li T, He Y, Wang J, Xie L, Qin X, Li S: Association between Tumor necrosis factor-alpha gene polymorphisms and prostate cancer risk: a meta-analysis. Diagn Pathol 2014, 9:74.

28. Wang Z, Zhang Y, Kong X, Li S, Hu Y, Wang R, Li Y, Lu C, Lin N, Chen W: Association of a polymorphism in PON-1 gene with steroid-induced osteonecrosis of femoral head in Chinese Han population. Diagn Pathol 2013, 8:186.

29. Dou K, Xu Q, Han X: The association between XPC Lys939Gln gene polymorphism and urinary bladder cancer susceptibility: a systematic review and meta-analysis. Diagn Pathol 2013, 8:112.

30. Siegel D, Yan C, Ross D: NAD(P)H: quinone oxidoreductase 1 (NQO1) in the sensitivity and resistance to antitumor quinones. Biochem Pharmacol 2012, 83:1033-1040.

31. Dinkova-Kostova AT, Talalay P: NAD(P)H:quinone acceptor oxidoreductase 1 (NQO1), a multifunctional antioxidant enzyme and exceptionally versatile cytoprotector. Arch Biochem Biophys 2010, 501:116-123.

32. Yu L, Chen J: Association of MTHFR Ala222Val (rs1801133) polymorphism and breast cancer susceptibility: an update meta-analysis based on 51 research studies. Diagn Pathol 2012, 7:171.

33. Guha N, Chang JS, Chokkalingam AP, Wiemels JL, Smith MT, Buffler PA: NQO1 polymorphisms and de novo childhood leukemia: a HuGE review and meta-analysis. Am J Epidemiol 2008, 168:1221-1232.

34. Zhang Y, Liu L, Tang Y, Chen C, Wang Q, Xu J, Yang C, Miao X, Wei S, Chen J, Nie S: Polymorphisms in TP53 and MDM2 contribute to higher risk of colorectal cancer in Chinese population: a hospital-based, case-control study. Mol Biol Rep 2012, 39:9661-9668.

35. Chao C, Zhang ZF, Berthiller J, Boffetta P, Hashibe M: NAD(P)H:quinone oxidoreductase 1 (NQO1) Pro187Ser polymorphism and the risk of lung, bladder, and colorectal cancers: a meta-analysis. Cancer Epidemiol Biomarkers Prev $2006,15: 979-987$

36. Lou $Y$, Li R, Xiong $L$, Gu A, Shi C, Chu T, Zhang X, Gu P, Zhong $H$, Wen S, Han B: NAD(P)H: quinone oxidoreductase 1 (NQO1) C609T polymorphism and lung cancer risk: a meta-analysis. Tumour Biol 2013, 34:3967-3979. 
37. Liu K, Tian H, Yu KZ, Shen WY, Mao ZC, Jin CH, Pan HB, He JX: Association between NQO1 Pro187Ser polymorphism and esophageal cancer: a meta-analysis. Tumour Biol 2013, 35:2063-2068.

38. Liu F, Luo L, Wei Y, Wang W, Li B, Yan L, Wen T: A functional NQO1 $609 C>T$ polymorphism and risk of hepatocellular carcinoma in a Chinese population. Tumour Biol 2013, 34:47-53.

39. Danson S, Ward TH, Butler J, Ranson M: DT-diaphorase: a target for new anticancer drugs. Cancer Treat Rev 2004, 30:437-449.

40. Ioannidis JP, Patsopoulos NA, Evangelou E: Uncertainty in heterogeneity estimates in meta-analyses. BMJ 2007, 335:914-916.

41. Xu Z, Yu L, Zhang X: Association between the hOGG1 Ser326Cys polymorphism and lung cancer susceptibility: a meta-analysis based on 22,475 subjects. Diagn Pathol 2013, 8:144.

doi:10.1186/1746-1596-9-100

Cite this article as: Peng et al:: The NQO1 Pro187Ser polymorphism and breast cancer susceptibility: evidence from an updated meta-analysis.

Diagnostic Pathology 2014 9:100.

\section{Submit your next manuscript to BioMed Central and take full advantage of:}

- Convenient online submission

- Thorough peer review

- No space constraints or color figure charges

- Immediate publication on acceptance

- Inclusion in PubMed, CAS, Scopus and Google Scholar

- Research which is freely available for redistribution 